\title{
MINIMAL-ACCESS MEDIAN STERNOTOMY FOR REPAIR OF CONGENITAL HEART DEFECTS
}

\author{
Giovanni Battista Luciani, MD, Caterina Piccin, MD, and Alessandro Mazzucco, MD, Verona, Italy
}

Minimally invasive approaches, including limited lateral thoracotomies, ${ }^{1}$ partial longitudinal or transverse sternotomies, ${ }^{2}$ and video-assisted thoracoscopic techniques, ${ }^{3,4}$ have recently been proposed for repair of congenital heart defects in an attempt to limit postoperative pain and respiratory dysfunction, allowing for prompt recovery, and to reduce the cosmetic impact of the scar. An alternative surgical technique is here reported, which combines the cosmetic advantages of the limited skin incision with those of complete median sternotomy.

Technique. A midline skin incision starting $2 \mathrm{~cm}$ below the angle of Louis and extending from a minimum of $3 \mathrm{~cm}$ (children 1 to 3 years) to a maximum of 7 $\mathrm{cm}$ (adults) in length is performed (Fig. 1). The soft tissues over the sternum are undermined to expose the suprasternal notch and the xiphoid appendage, and a median sternotomy is completed with the use of an oscillating saw. The pericardium is entered through a right paramedian vertical incision and traction sutures are placed only on the right free edge of the pericardium so as to enhance exposure of the venae cavae and the interatrial groove. The ascending aorta and the superior vena cava (SVC) are cannulated and normothermic cardiopulmonary bypass is established, thereby emptying the heart. The junction of the atrium and inferior vena cava (IVC) is developed and the IVC purse-string suture is put in place. The IVC cannula is tunneled through a separate 1 to $1.5 \mathrm{~cm}$ long subxiphoid midline skin incision and complete bypass is begun. Both the IVC purse-string suture and the snare tourniquets are tunneled from the pericardium through the incision along with the cannula, so as to lie outside the narrow operative field (Fig. 2). For ventricular septal defect repair, a vent line can be inserted through the right superior pulmonary vein. The aorta is then crossclamped and the heart arrested with cardioplegic solution injected into the aortic root. The intracardiac repair is performed via a standard right atriotomy. After deairing of the heart via the aortic root, the clamp

From the Division of Cardiac Surgery, University of Verona, Verona, Italy.

Received for publication Feb. 4, 1998; accepted for publication March 5, 1998.

Address for reprints: Giovanni Battista Luciani, MD, Division of Cardiac Surgery, University of Verona, O.C.M. Piazzale Stefani 1, Verona, 37126 Italy.

J Thorac Cardiovasc Surg 1998;116:357-8

Copyright (c) 1998 by Mosby, Inc.

$0022-5223 / 98 \$ 5.00+0 \quad \mathbf{1 2 / 5 4} / \mathbf{9 0 1 9 0}$ is released and the heart is defibrillated. The IVC cannula can also be removed during weaning from extracorporeal circulation, still allowing for adequate exposure of the cannulation site. The subxiphoid skin incision, which served as "port" for the cannula, is now used to insert the pericardial chest tube. The sternotomy is closed with stainless steel wires and the soft tissues with running absorbable suture.

During the past 6 months, this technique was used in 18 patients with a median age of 6 years (range 1 to 47 years) and a weight of $24 \mathrm{~kg}$ ( 9 to $70 \mathrm{~kg}$ ) for repair of atrial septal defect (10 primary, 5 patch), sinus venosus defect with partial anomalous pulmonary venous connection (2 patients), and ventricular septal defect (1 patient). The median skin incision length was $4 \mathrm{~cm}$ (range 3 to $7 \mathrm{~cm}$ ) and the median aortic crossclamp and operative times were 18 minutes (range 7 to 38 minutes) and 2.4 hours (range 2.0 to 3.1 hours), respectively. All patients were extubated in the operating room and sent to the recovery department. Median hospital stay was 3 days (range 2 to 4 days). There were no major complications, such as respiratory failure or infection, hemorrhage necessitating reexploration or blood transfusion, or circulatory instability. No instances of wound infection or dehiscence were observed at follow-up. One 12-year-old girl with a family history of congenital heart disease (mother and younger sister operated on for atrial septal defect) and of skin cheloid (mother and sister with cheloid of the skin incision) had a cheloid of the wound.

Comment. Elective repair of simple congenital cardiac defects is feasible via lateral thoracotomy, ${ }^{1}$ partial sternotomies, ${ }^{2}$ and minithoracotomies with video-assisted thoracoscopy. ${ }^{3,4}$ When compared with standard median sternotomy, notable disadvantages of minimally

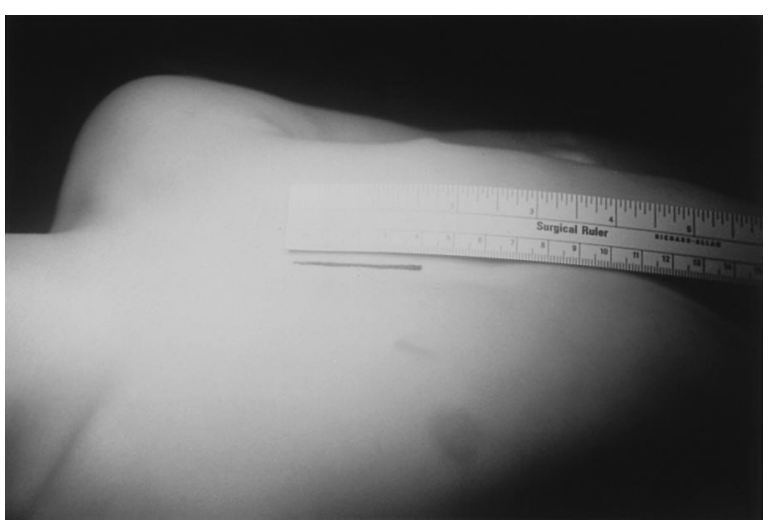

Fig. 1. Length $(4 \mathrm{~cm})$ and position of the midline skin incision in a 4-year-old patient. 


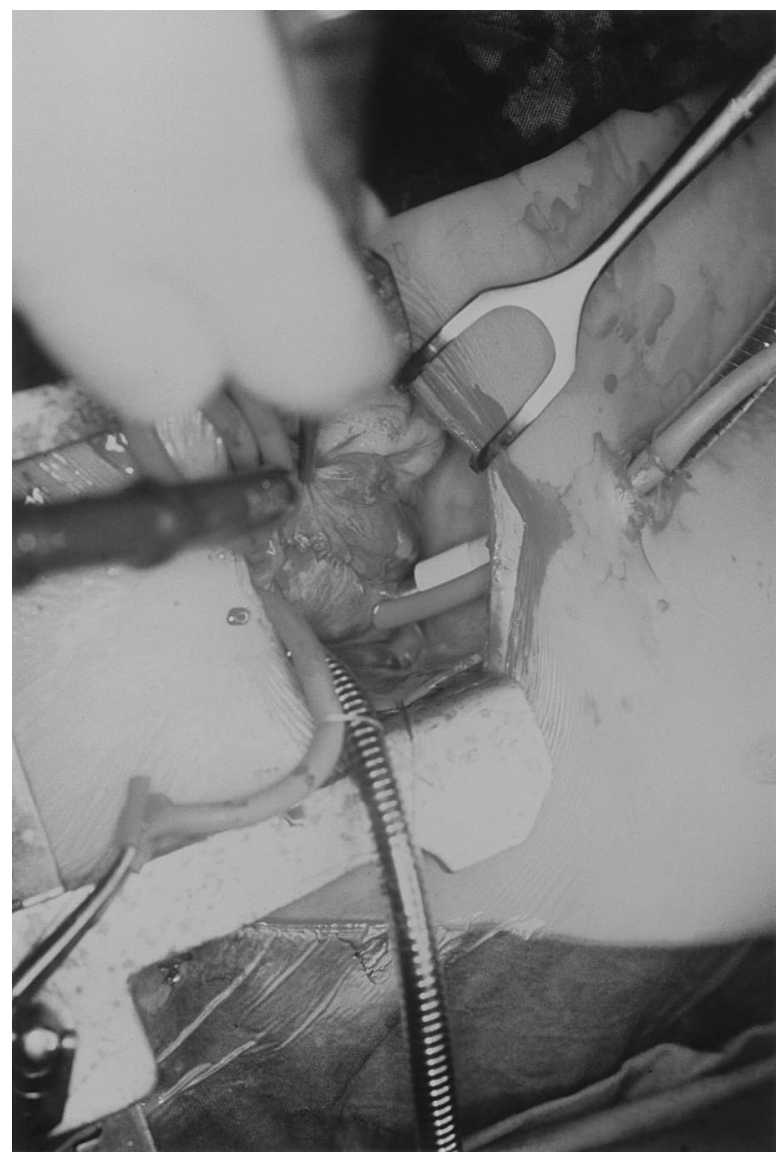

Fig. 2. Operative view after completion of the right atriotomy suture line. The arterial and right-angled, metaltipped SVC cannulas are seen on the right-hand side of the picture. Note the technical detail relative to the right-angled, metal-tipped IVC cannula, which, along with its tourniquet, has been tunneled through a separate subxiphoid port. This incision will later host the chest tube for pericardial drainage.

invasive approaches include sensitive denervation of the breast and greater incisional pain for the lateral thoracotomy, need for inguinal vessel cannulation and limited exposure for the minithoracotomy with video- assisted techniques, and unstable sternal reconstruction for the partial sternotomies. Most important, the results thus far reported in terms of postoperative recovery are no better than the standards set by repair via median sternotomy. ${ }^{3-5}$

The approach here reported consists in a minimal skin incision with a complete median sternotomy. Because the extent of the skin incision is the primary difference with the standard approach, it raises the "philosophical" question of what a minimally invasive technique truly consists of. Indeed, if the postoperative recovery pattern is comparable with that of the other less invasive access routes, a minimal skin incision with a complete median sternotomy ought to be defined as "minimally invasive." Our experience confirms that this technique offers satisfactory cosmetic results, stable sternal reconstruction, minimal interference with respiratory mechanics, and minimal pain, allowing for extubation in the operating room and speedy recovery. Because exposure of the ascending aorta, SVC, and right atrium are prompt and because the IVC can be cannulated during partial cardiopulmonary bypass by way of an accessory subxiphoid route, a variety of congenital heart defects (including ostium secundum, sinus venosus, atrioventricular septal defects, and ventricular septal defects) can be repaired. Conversion to a more extended incision in case of intraoperative incidents or unexpected anatomy is immediate, resulting in no cosmetic mutilation or chest instability. A minimalaccess complete sternotomy approach may represent a simple, rapid, low-risk, and low-cost alternative to partial sternotomies or minithoracotomies for repair of congenital cardiac lesions.

\section{REFERENCES}

1. Rosengart TK, Stark JF. Repair of atrial septal defect through a right thoracotomy. Ann Thorac Surg 1993;55:1138-40.

2. Moreno-Cabral RJ. Mini-T sternotomy for cardiac operations [letter]. J Thorac Cardiovasc Surg 1997;113:810-1.

3. Chang $\mathrm{CH}$, Lin PJ, Chu JJ, et al. Video-assisted cardiac surgery in closure of atrial septal defect. Ann Thorac Surg 1996;62:697-701.

4. Lin PJ, Chang $\mathrm{CH}$, Chu JJ, et al. Minimally invasive cardiac surgical techniques in the closure of ventricular septal defect an alternative approach. Ann Thorac Surg 1998;65:165-70.

5. Mavroudis C. VATS ASD closure: a time not yet come. Ann Thorac Surg 1996;62:638-9. 